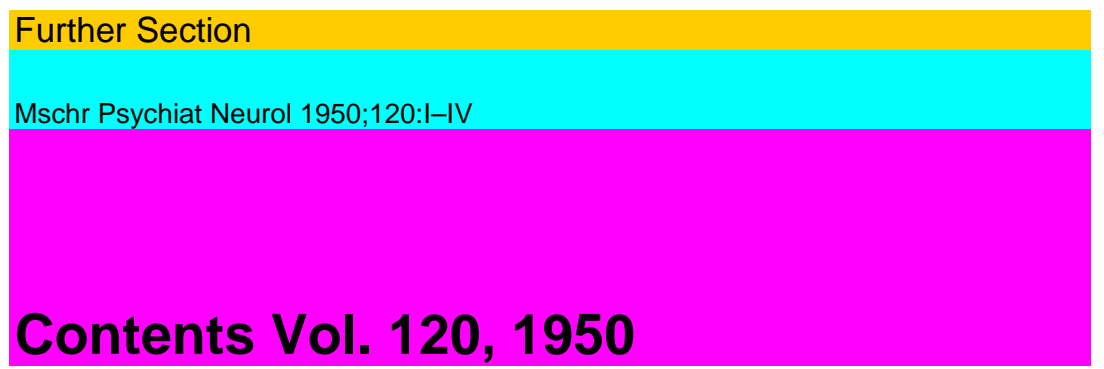

Monatsschrift für

\title{
Psychiatrie und Neurologie
}

Revue Mensuelle de Psychiatrie et de

Neurologie Monthly Review of Psychiatry and

Neurology

L.VAN BOGAERT,Anvers B.BROUWERf, Amsterd. E. CARP, Leiden 0. DIETHELM, New York R. GJESSING, Oslo E. GRÜNTHAL, Bern H. HELWEG, Kopenhagen COLLABORATORES:

B. JACOBOWSKY, Upsala E. KAHN, New Haven G. LANGFELDT, Oslo J. LEPINE, Lyon A. LEWIS, London J. LHERMITTE, Paris
A. MEYER f, Baltimore
F. MOREL, Geneve
G. PAMBOUKIS, AthenH. SCHOU, DianalundJ. STAEHELIN, BaselH. STECK, Lausanne
C. I. URECHIA, Sibiu

EDITOR:

J. KLAESI, Bern 


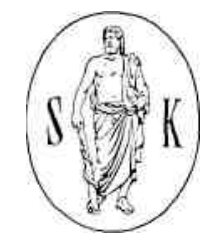

Vol. 120

1950

BASEL (Schweiz)

S. KARGER

NEW YORK

Printed in Switzerland

Alle Rechte vorbehalten Tous droits reserves

Druck von Gasser und Cie. AG. in Basel

Clichgs: Ab er egg-Steiner \& Cic. AG. Bern

Printed in Switzerland

INDEX

Barraquer-Bordas, L.: De la nature clinique des reflexes dits „profonds" et de ce que l'on appelle leur „zone reflexogene". 
Barraquer-Bordas, $L$. viele Castaner, $E$.

Bogaert, L. van: XVUL Sur les secousses cloniques spontanees et intentionnelles dans des paralysies generales atypiques ....

Bogaert, L. van: XJX.. Sur une athetose double (etat marbre) chez un frere et une soeur.

Bogaert, L. van et G. Tverdy : Alterations du Systeme nerveux central dans l'avitaminose E chez l'homme

Castaner, E., C. Oliveras de la Riva and L. Barraquer-Bordas: Primitive Melanoma of the Cauda Equina

Celestino da Costa, A.: Contribution ä l'etude de la cytochimie de la differenciation neurale.

310

Cobb, S.: Treatment of Psychoneurosis and Results of Follow-up Study ... 316

Dimitrijevic, D. T.: Zur Struktur und Pathophysiologie des Thalamussyndroms

Gäl, P.: Contribution to the Clinic and Psychopathology of the Non-Epileptic

Poriomany.....

206

Gereb, T.: Die Beziehungen der nekrotisierenden Myelopathie zur. multiplen

Sklerose.

237

Glees, P. und E. Zander: Der Tractus tegmento-oliyaris des Menschen (nach zwei Fällen von Mittelhirntraktotomien)

Gloor, $P$. vide Woringer, $E$.

Gousenberg, M.: Contribution ä l'etude des troubles psychiques du mercurialisme chronique.

Gozzano,M.: Die Autoallergie in der Pathologie des Nervensystems.

Grünthal, E.: Beiträge zur Kenntnis des extra-pyramidal-motorischen Systems

Heimann, H.: Der Einfluß von Karl Jaspers auf die Psychopathologie ...

Horst, L. van der: Lebensreichtum und Lebensalter

Hufschmidt, $H$. vide Vonwiller, $P$.

Juhasz, P.: ContriLutions to the Clinical Aspects and to the Patho-Anatomy of Pachymeningitis Spinalis Externa 152

Morel, F.: Petite contribution ä l'etude d'une angiopathie apparemment dyshorique et topistique.

352

Oliveras de la Riva, C. vide Castaner, E.

Riese, W.: Neuro-Pathology of Mental Disorders

Rohracher, H.: Skizze einer physiologischen Gedächtnistheorie .

Rose, J. E.: Some Modern Aspects of the Functional Anatomy of the Thalamus

Schiller, E.: Kristalleinschlüsse in menschlichen Zellkernen

Simma, K.: Zur Cytoarchitektonik des menschlichen Centram medianum thalami

Sole-Sagarra, J.: Contribution ä l'etude de la choree de Huntington et de la paralysie agitante.

Sorgo, W.: Die gegenseitigen Abhängigkeitsverhältnisse topistischer Einheiten (C. und 0. Vogt) in der Neurochirurgie.

Tverdy, G. vide Bogaert, L. van

Spiegel, E. A., H. T. Wycis and C. W. Umlauf: Electroencephalographic Studies before and after Thalamotomy.

Umlauf, C. W. vide Spiegel, E. A.

Vonwiller, P. und H. Hufschmidt: Die Nigrosinfärbung der Hirnrinde . . . . 
Vujic, V.: Larvate Encephalitis and a New Extrapyramidal Syndrome . . .

Wartenberg, R.: Pathophysiology of the Hemifacial Spasm ...

Woringer, E. et $P$. Gloor: Existence simultanee de deux tumeurs melaniques

du nevraxe, l'une medullaire, l'autre cerebrale......

Wycis, H. T. vide Spiegel, E. Ä.

Zander, E. vide Glees, $P$.

Relata ..

282

Libri .

N. Necrologia

294

Corrigenda

104

103, 292, 434 